infection. In young individuals, as in the case of the junior hospital doctors discussed in my article, previous $B C G$ vaccination should not be considered an adequate explanation for a strongly positive tuberculin reaction. Superinfection with Mycobacterium tuberculosis is the more likely cause. In other cases, however, a strongly positive tuberculin reaction may be due to either healed primary or adult tuberculosis; in both tuberculin hypersensitivity may remain high for many years." Dr Gatley's survey of tuberculin reactions cannot be interpreted without further information, including the details mentioned above.

Freda Festenstein

London Chest Hospital,

London E2 9JX

1 Festenstein $F$. Lymphangitis after tuberculin tests. Br Med F 1984;289:625.

aplin M. The tuberculin test in clinical practice.
London: Bailliere Tindall, 1980 .

\section{Design and interpretation of clinical trials}

SIR,-Dr Stuart J Pocock (5 January, p 39) has expertly reaffirmed some of the statistical hazards which continue to be encountered in clinical research. It is regrettable that there is still a need for such articles to be written and rather disappointing that $\mathrm{Mr} \mathrm{A} \mathrm{V}$ Pollock and Ms Mary Evans (19 January, p 243) have responded in a way which helps to perpetuate the concept of a "magic" arbitrary probability level of rejecting null hypotheses.

Dr Pocock wrote of the "magic" of the $\mathrm{p}=$ 0.05 barrier. Mr Pollock and Mary Evans have suggested that null hypothoses should be rejected only if this threshold is crossed even after one adverse change is artificially injected into the data; by so doing, they are merely redefining their magic point as slightly less than 0.05 . While creditable as a move towards higher statistical confidence, such a procedure merely represents an arithmetical, rather than a conceptual, change. It would be more helpful to forget arbitrary thresholds and simply quote the actual probability level or confidence limits.

A second example of magic also shows itself, in this case with some help from statisticians. Although confidence intervals can be a useful way of presenting statistical results, there is no truth in the almost universal implication that they convey different information from probability levels. With two sided testing, $95^{\circ}$. confidence limits have the same relevance as a $\mathrm{p}=0.05$ rejection threshold. As illustrated by Mr Pollock's figures, this means that a difference only just achieving $p<0.05$ will inevitably have a $95^{\circ}$, confidence interval which only just fails to include zero and which extends up to nearly twice the observed difference. Only when $p$ values are much lower than the rejection threshold will the confidence interval based on this threshold-for example, $95^{\circ}$ limits for $\mathrm{p}=0.05-$ become narrower and more remote from zero. If we stubbornly retain arbitrary thresholds for determining significance we must at least be consistent and refrain from criticising results which only just achieve our criteria, whether expressed by $\mathrm{p}$ value or by the corresponding confidence interval. Those who criticise in this way are effectively saying that the standard magic threshold should be lower than 0.05 , a suggestion which is perhaps worthy of debate.

Virtually all these problems, and their associated magic, could be avoided if arbitrary thresholds of significance were abandoned in favour of stated probability levels, leaving the individual reader to make his own interpretation of $\mathrm{p}$ values such as 0.051 and 0.049 .

\section{J R WhitTingtoN}

Harrow, Middlesex HA2 7AJ

Sir,-Mr A V Pollock and Ms M Evans seem worried that if they were to transfer an event from one arm of their trial to the other the significant difference would disappear. But if they did it the other way the significant difference would increase. The fact is, we have to rely on what we observe.

There is, however, another way of looking at their data which is more pragmatic: the likelihood ratio. The difference in favour of cephaloridine over ampicillin was $6.5^{\circ}{ }_{n}$. The standard deviation of this difference is $3.2^{\circ} \mathrm{o}$. By referring to tables of the area of the normal curve, it is possible to assign probabilities to observing differences of greater than $6.5^{\circ},(p=0.5)$ and less than $0(p=0.2)$. The odds in favour of the difference being equal to or greater than $6.5^{\circ}$, compared with nothing or less, are $25: 1$. On this interpretation I would prefer to use cephaloridine. The advantages of this technique are that one does not have to use rigid criteria for acceptance, and that any level of difference can be assessed. The method is analogous to the use of receiver operating characteristic curves in the interpretation of laboratory tests. ${ }^{1}$ Justifiable concern has been expressed about the failure of many so called negative trials to exclude the possibility of a real difference between treatments. ${ }^{2}$ By the use of the calculations described here clinicians can decide quite simply whether they think the odds of success justify the use of the treatment.

Royal Devon and Exeter Hospital (Wonford),

G H HALL

\section{Swetz JA, Pickett RM. Evaluations of diagnostic systems. New York: Academic Press, 1982 \\ 2 Freiman JA, Chalmers TC, Smith H, Kubler RR. The importance of beta, the tipe II error and sample size in the design and interpretation of the clinical trial. $N$ Engl f Med 1978;299:690.}

SIR,-As general practitioners with a major involvement in clinical trials we read $\mathrm{Dr}$ Stuart J Pocock's article with interest. We would disagree with one point-that minimising the treatment difference in prognostic factor distributions increases the administrative burden. Using Taves's minimisation technique $^{1}$ with a programmable calculator we have achieved closely matched groups in several studies. This balance of clinical variables is important with the small numbers usually involved in general practice studies. The method has proved cheap and simple to administer. It also has the added advantage that by involving an independent third party the investigator can not only maintain a double blind design but is fed an allocation number which does not tell him the patient's treatment group. In our first study with this method we balanced 52 patients for 19 cells

JAMES HOSIE

Glasgow G13 2SW GILIIAN Hosie

1 Taves DR. Minimization: a new method of assigning patients to treatment and control groups. Clin
Pharmacol Ther $1974 ; 15: 443-53$.
SIR,-Dr Stuart J Pocock (5 January, p 39) has chosen to illustrate his section on "Chaos caused by non-randomised trials" by reference to the studies by the group associated with us on vitamin supplementation for prevention of neural tube defects. Unfortunately, he has chosen to quote our earlier publications, and those not entirely accurately.

Firstly, the control group did not include any mothers who had elected not to take supplements. Secondly, our most recently published figures showed three neural tube defects in 429 examined offspring of fully supplemented mothers and 24 neural tube defects in 510 offspring of unsupplemented mothers. ${ }^{1}$ Thirdly, while it is true that there was an excess of control mothers from Northern Ireland in our first cohort, this was not true of the second. ${ }^{1}$ Furthermore, a significant protective effect of vitamins has been shown in Northern Ireland, using data which included mothers in the first two cohorts. ${ }^{2}$ There were three neural tube defects in 226 examined offspring of fully supplemented mothers and 14 neural tube defects in 293 offspring of unsupplemented mothers.

Nevertheless, we share, and have often expressed the view that the original trial should have been randomised. We hope Dr Pocock has read our papers sufficiently carefully to appreciate that his remarks are directed (in this case) to research ethics committees and not to the investigators.

$\mathrm{R}$ W SMITHELLS SHULA SHEPPARD

JENNIFER WILD

C J SCHORAH

MARY J SELler

Paediatric Research Unit,

Guy's Hospital Medical School, London SE1 9RT

Countess of Chester Hospital,
Chester

D W FiELdiNG

1 Smithells RW, Nevin NC, Seller MJ, et al. Further experience of vitamin supplementation for prevention of neural tube defect recurrences. Lancet 1983 ; tion of neura

2 Seller MJ, Nevin NC. Periconceptional vitamin supplementation and the prevention of neural tube defects in south-east England and Northern Ireland.
J Med Genet 1984;21:325-30.

\section{Trimethoprim resistance in Gram negative urinary pathogens}

SIR,-Dr Rosalind Maskell (12 January, p 156) still seems to be under the impression that the addition of a sulphonamide to trimethoprim discourages the development of trimethoprim resistance during clinical use. Yet not a single supporting fact is presented. Firstly, fluctuations in the incidence of resistance are small; secondly, no data are given on the total consumption of trimethoprim, co-trimoxazole, or other antibiotics, notably the ampicillins, that might affect the incidence of resistance. Ratios of antibiotic use have limited meaning, but if these were thought to be important then the proportion of trimethoprim to cotrimoxazole has actually increased during 1981-4 from $1: 11$ to $1: 6$ in two areas that refer specimens to Dr Maskell's laboratory. It could be argued therefore that the use of trimethoprim is increasing yet resistance is decreasing.

In the Leeds General Infirmary, a large teaching hospital, trimethoprim replaced cotrimoxazole in early 1984. In the enterobacteriaceae the incidence of resistance to 
trimethoprim has remained the same $(26 \%)$ in the six months before the change and in the six months afterwards. Resistance to sulphonamides has dropped from $40 \%$ to $37^{\circ}$. Between 1983 and 1984 the total amount of trimethoprim prescribed in the hospital increased by $31 \%$. These findings are in accord with our previous data that there is no evidence that adding a sulphonamide to trimethoprim discourages resistance.

We would like to invite Dr Maskell to perform the following experiments: select cultures of either fully sensitive or sulphonamide resistant (trimethoprim sensitive) enterobacteriaceae, expose them to trimethoprim of the sort of concentration-for example, $256 \mu \mathrm{g} / \mathrm{ml}$ - to which strains are highly resistant, and see if any resistant variants can be selected. She will find few such variants from either type of culture, and the few bacteria that may struggle through will be grossly defective and probably not pathogenic.

Surely the time has come to withdraw cotrimoxazole for urinary and many other infections, such as typhoid. The major active component of co-trimoxazole is trimethoprim. It is effective and safe. It should be used alone unless reliable evidence is presented that sulphonamides have a contribution. Dr Maskell provides no such data.

R W LACEY R G LOKER

Department of Microbiology, University of Leeds,
Leeds LS2 9J T

J COOKE

Department of Pharmacy,

Leeds Western Health Authority,

Leeds

SIR,-Dr Rosalind Maskell postulated that the decline in resistance to trimethoprim resulted from a decrease in the use of trimethoprim alone as compared with cotrimoxazole. The rationale behind this may $\mathrm{R} T$ CALVERT

use of plain trimethoprim must be considered only in the context of the far greater amount of co-trimoxazole used overall. Any alteration in the pattern of resistance to trimethoprim is surely more likely to result from co-trimoxazole.

Medical Department,

Southampton SO3 $3 \mathrm{JD}$

1 Brumfitt W, Hamilton-Miller JMT, Wood A. Evidence for a slowing in trimethoprim resistance during $1981-$ a comparison with earlier years. $\mathcal{J}$ Antimicrob Chemother 1983;11:503-9.

2 Huovinen P, Mantyjarvi R, Toivanen P. Trimethoprim $\mathrm{res}$

3 Knothe $\mathrm{H}$. The effect of trimethoprim-sulphonamide, trimethoprim and sulphonamide on the occurrence trimesioprim and sulphonamide on the occurrence of resistant enterobacteriaceae in hu
flora. Infection $1979 ; 7$ (suppl 4):321-3.

4 Amyes SGB. The detection and incidence of transferable trimethoprim resistance. Health Bulletin 1983;41:99-107.

SIR,-The letter from Dr Rosalind Maskell (12 January, $p$ 156) has prompted us to look at the resistance to trimethoprim of Enterobacteriaceae causing acute urinary tract infections in family practice in north Hampshire.

Since 1975 this laboratory has tested and reported trimethoprim and sulphonamide sensitivities separately. Between 1976 and 1980 intermittent studies of these organisms were made, and "best guess" antimicrobials recommended to general practitioners in our area. Since March 1980 trimethoprim alone has been recommended. From June 1983 information about these organisms has been stored on microcomputer discs. The results are summarised in the table. The columns for co-trimoxazole and trimethoprim are practitioners' prescription intentions for patients who subsequently proved to have significant bacteriuria; the drugs include both generic and proprietary preparations.

We suggest that, in spite of a large increase in the use of trimethoprim alone, there has

Resistance to trimethoprim of Enterobacteriaceae from acute urinary tract infections in family practice and the prescribing intentions of general practitioners

\begin{tabular}{|c|c|c|c|c|}
\hline & \multirow{2}{*}{$\begin{array}{c}\text { Total } \\
\text { No of } \\
\text { organisms }\end{array}$} & \multirow{2}{*}{$\begin{array}{l}\text { No }\left({ }^{\prime}{ }^{\prime \prime}\right) \\
\text { resistant to } \\
\text { trimethoprim }\end{array}$} & \multicolumn{2}{|c|}{ No of prescriptions for: } \\
\hline & & & Co-trimoxazole & Trimethoprim \\
\hline $\begin{array}{l}1976 \text { (Feb-Mar) } \\
1980 \text { (Mar-Jul) } \\
1983 \text { (Jul-Dec) } \\
1984 \text { (Jan-Jun) } \\
1984 \text { (Jul-Dec) }\end{array}$ & $\begin{array}{l}297 \\
254 \\
849 \\
787 \\
797\end{array}$ & $\begin{array}{r}9(3 \cdot 0) \\
19(7 \cdot 5) \\
39(4 \cdot 6) \\
37(4 \cdot 7) \\
43(5 \cdot 4)\end{array}$ & $\begin{array}{r}57 \\
123 \\
93 \\
96\end{array}$ & $\begin{array}{r}51 \\
276 \\
265 \\
357\end{array}$ \\
\hline
\end{tabular}

be unsound and is not supported by other published studies. ${ }^{12}$ There is evidence that the sulphonamide component does not prevent trimethoprim resistance developing when co-trimoxazole is used. The use of either trimethoprim or co-trimoxazole has been shown to remove faecal coliforms from the gut flora, which return, fully sensitive, when treatment is discontinued. The use of a sulphonamide alone, however, quickly selects resistant faecal coliforms. ${ }^{3}$ Furthermore, the discussion should not be confined to these two drugs, the transfer of plasmids conferring both ampicillin and trimethoprim resistance being the probable explanation for a concurrent increase in ampicillin usage and trimethoprim resistance in some hospitals. ${ }^{4}$

Dr Maskell reports the ratio of the use of co-trimoxazole to trimethoprim at St Mary's Hospital, Portsmouth as being $16: 1$ in 1982 and $19: 1$ in 1984. This small reduction in the prim resistance. Therefore we shall continue to recommend the use of trimethoprim alone in this situation, particularly to prevent patients receiving an unnecessary sulphonamide.

P A Boswell

Microbiology Department, Basingstoke District Hospital,
Basingstoke, Hants RG24 9NA SUSAN HINDER

\section{Prevention of meningitis in head injury}

SIR,-We were surprised at Dr Sanderson's suggestion (24 November 1984, p 1456) that chloramphenicol might supersede sulphonamides in preventing meningitis in fractures of the base of the skull. While toxicity from chloramphenicol is uncommon, the serious
Duphar Laboratories Ltd

ness and irreversibility of such toxicity would discourage many from using it in other than life threatening infections. Our experience and considerations of bacterial sensitivity and antibiotic pharmacokinetics suggest that the use of chloramphenicol is not justified.

Although this bony injury is relatively common ( 50 such cases having been admitted to Bristol Road Infirmary in 1983), with our standard prophylaxis of sulphonamide plus benzylpenicillin no case of meningitis secondary to such an injury has been detected in our laboratory in the past five years. Thus we are faced with a recommendation to change a regimen that seems to work well, on the grounds of changing patterns of bacterial antibiotic resistance.

Our reading of Dr Sanderson's references on antibiotic resistance suggests very different figures from those in his table. For Haemophilus influenzae he appears to have extracted the resistance rates for chloramphenicol and sulphonamide $\left(8^{\circ} \circ\right.$ and $51^{\circ} \%$ respectively) from table I of his reference. ${ }^{1}$ This table deals with a selected group of strains which showed reduced inhibition zones to any of the antibiotics tested. In the text the overall resistance rates were concluded to be $1.03^{\circ}$, for chloramphenicol and $1.5^{\circ}$ for sulphonamide. In considering Streptococcus pneumoniae, ${ }^{2} \mathrm{Dr}$ Sanderson sets a breakpoint for resistance ( $>2 \mathrm{mg} / \mathrm{l}$ ) which appears unreasonably low, in that all the strains tested were inhibited by $4 \mathrm{mg} / 1$ of chloramphenicol and by $8 \mathrm{mg} / 1$ of sulphonamide, and these concentrations are readily surpassed in serum and in cerebrospinal fluid after standard doses whether or not the meninges are inflamed.

If it is felt that antibiotic cover must be extended to include reliably Staphylococcus aureus we suggest that trimethoprim (which also achieves therapeutically effective concentrations in cerebrospinal fluid and to which almost all strains of the three pathogens are sensitive) may be added to the sulphonamide (as co-trimoxazole) in prophylaxis; cefuroxime alone is another possibility. At present, however, there seems to be little evidence of a need to change a satisfactory prophylactic regimen.

M J KeLLY

J H Paton

Bristol Royal Infirmary,

Bristol BS2 8HW

1 Philpott-Howard J, Williams JD. Increase in antibiotic resistance in Haemophilus influenzae in the United Kingdom since 1977: report of study group.

2 Cooksey RC, Facklam RR, Thornsberry C. Antimicrobial sensitivity patterns of Streptcooccus pneumon

3 Barling RWA, Selkon JB. The penetration of antibiotics into cerebrospinal fluid and brain tissue. $f$ Antimicrob Chemother $1978 ; 4: 203-27$.
f

\section{How soon after myocardial infarction} should plasma lipid values be assessed?

SIR, - We have read with interest the article by $\operatorname{Dr} R \mathrm{E} J$ Ryder and others (15 December, p 1651) about the evolution of serum lipid values during the acute phase of myocardial infarction and three months later. We have measured serum lipid values in 12 men with mvocardial infarction. Sampling was performed on admission within 12 hours after the onset of symptoms and $2,4,6,8,10$, and 30 days after admission. The sample on the 30 th day was obtained at an outpatient clinic two weeks after discharge. Contrary to the results of Dr Rvder and others, we ohserved 